\title{
Clinical, Epidemiological and Laboratory Profile of Dengue Fever at Tertiary Care Hospital in Hyderabad, India
}

\author{
Qursheed Sultana ${ }^{1 *}$, Mohammed Abdur Rab Ansari², Mahwish Jawaid ${ }^{1}$, \\ Mohd Ahmed $^{1}$, Khaled Ahmed ${ }^{1}$ and Maimoona Mustafa ${ }^{1}$ \\ ${ }^{1}$ Deccan College of Medical Sciences, Hyderabad, Telangana, India \\ ${ }^{2}$ MBBS Deccan, College of Medical Sciences, India \\ *Corresponding author
}

\section{A B S T R A C T}

\section{Keywords}

Epidemiological,

Laboratory profile,

Dengue fever, Tertiary care hospital

\section{Article Info}

Accepted:

18 September 2018

Available Online:

10 October 2018
Dengue Fever (DF), Dengue hemorrhagic fever (DHF) and Dengue Shock Syndrome (DSS) are topmost public health concerns today, especially in tropical and subtropical countries, mainly involving urban and semi-urban areas. In India, Dengue is hyperendemic (Category A) and is a notifiable infectious disease. Though gaps exist in terms of public health measures and health education among public, microbiological laboratory plays a crucial role in confirmation of dengue infection and estimation of burden of disease. The aim of the study was to investigate and assess the epidemiology and prevalence of dengue infection in patients attending our tertiary care hospital in Hyderabad. A total of 1381 patients of suspected Dengue presented during the study period of January - December 2017 were included in the study. All the samples were processed using Dengue NS1 microlisa, Dengue MAC IgM ELISA and Dengue GAC IgG ELISA. Out of these 1381 samples, 395 were serologically positive; 240 (61\%) for NS1 antigen and $82(21 \%)$ for anti-dengue $\operatorname{IgM}$ and IgG antibodies. Maximum number of dengue positive serum samples was observed in the month of November $421(31 \%)$ followed by October $310(23 \%)$. Out of 395 positive dengue cases, 309 were adult and 86 were of pediatric age group. Majority of positive cases falls under age group of 11-20 years 149(38\%) followed by 21-30 years of age group 104 (26\%). The lowest age of positive case was 4 months and highest was 71 years. Most common clinical presentation was fever followed by thrombocytopenia and arthralgia. To look for platelet and leucocyte counts, blood samples were drawn for routine hemogram at the time of admission. This was evaluated by fully automated haematology analyser-5 part differential. Thrombocytopenia was defined as platelet counts less than 1,50,000/microliter and confirmed on peripheral smear examination. Leucocyte counts of dengue patients were recorded and in majority of patients they were in normal range (400011000 per microliter). From the above study, it is concluded that prompt surveillance and regular awareness are the two major steps to be taken for early detection and prevention of dengue virus infection.

\section{Introduction}

Dengue (DEN) is the most rapidly spreading viral disease in the world with a 30 - fold increase in incidence in the last 50 years. An estimated 50 million DEN infections occur annually and approximately 2.5 billion people live in DEN endemic countries (World Health Organization, 2009). In India, Dengue is hyperendemic (Category A) and is a notifiable 
infectious disease (Park, 2013). Since DEN produces a broad-spectrum of symptoms, a diagnosis based only on clinical symptoms is unreliable. Early laboratory confirmation is valuable because some patients may deteriorate rapidly resulting in death (World Health Organization, 2009). During primary infection, IgM appears after 5-6 days and IgG after 7-10 days. During a secondary infection, high levels of IgG are detectable even during the acute phase and rise over the next 2 weeks, whereas IgM are low or even absent in some cases of secondary infection. IgM antibodies suggest a recent infection; however, they can persist for 2-3 months (Guzmán and Kourí, 2002; De Paula and Fonseca, 2004). High titres of $\mathrm{IgG}$ are a criterion of secondary infection. Viral nonstructural 1 (NS1) antigen is abundant in the serum of patients in the early stages of DEN infection, lasting from 1 to 9 days; therefore, NS1 antigen ELISA, especially when used together with a IgM capture ELISA, is sufficiently informative in an endemic setting (Blacksell et al., 2008).

\section{Materials and Methods}

This prospective study was carried out in the microbiology laboratory between January 2017 and December 2017 and was approved by the Institutional Ethics Committee of Deccan College of Medical Sciences. Case inclusion criteria included presentation of sudden high fever $\left(39-40^{\circ} \mathrm{C}\right)$ of 2-7 days duration, intense headache, myalgia, arthralgia or rash. The case was excluded if there was evidence of bacterial or other viral illness based on laboratory testing, duplicate samples were excluded. All cases were managed according to WHO Guidelines (National Guidelines for Clinical Management of Dengue Fever, 2015). After consent of the patient, blood was taken by venipuncture according to standard guidelines. Serum was refrigerated $\left(2-8^{\circ} \mathrm{C}\right)$ if not tested within two days. Sera showing haemolysis, icterus, lipaemia or microbial growth were excluded because they may cause false positive / negative interpretation. Before beginning assay, all reagents were brought to room temperature $\left(20-25^{\circ} \mathrm{C}\right)$. Blood (serum) samples collected from the patients were processed using $\operatorname{IgM}$ and $\operatorname{IgG}$ capture ELISA and NS1 ELISA according to manufacturer's instructions (J. Mitra and Co. Pvt. Ltd.). In this kit, wells were coated with purified dengue virus type 2 antigen cultured in Vero cells. The test was executed according to the manufacturer's instructions. Dengue NS1 antigen, if present in serum, binds to anti-NS1 antibodies attached to the polystyrene surface of the micro-wells which is followed by chromogenic reaction. Development of colour was indicative of the presence of dengue NS1 antigen in the test sample. An active primary dengue infection was considered when positive result came (>11 Panbio Units). IgM antibodies in the patient's blood was captured by Antihuman IgM ( $\mu$ chain specific) coated on the wells. In the next step, DEN antigen was added which could capture IgM, if IgM and antigen were homologous. Unbound antigen was removed during the washing step. In the subsequent step Biotinylated flavivirus cross-reactive monoclonal antibody (Hx-B) was added followed by Avidin -HRP. Subsequently, substrate/chromogen was added and noted for development of colour. The reaction was stopped by $1 \mathrm{~N} \mathrm{H} 2 \mathrm{SO} 4$. Intensity of colour/optical density was monitored at 450 $\mathrm{nm}$. For quality control, each kit had a positive and a negative control. Calculations and interpretation were done as per the kit literature.

\section{Results and Discussion}

A total of 1381 patients of suspected Dengue presented during the study period. Out of these 1381 samples, 395 were serologically positive by at least one of the following tests-IgM ELISA, IgG ELISA, NS1 ELISA (Figure 1). 
Maximum number of dengue positive serum samples were observed in the month of November $421(31 \%)$ followed by October $310(23 \%)$ (Table 1).

Out of 395 positive dengue cases, 309 were adult and 86 were of pediatric age group (Figure 2). Majority of positive cases falls under age group of $11-20$ years $n=149(38 \%)$ followed by 21-30 years of age group $n=104$ (26\%) (Figure 3). The lowest age of positive case was 4 months and highest was 71 years.

Most common clinical presentation was fever followed by thrombocytopenia and arthralgia. To look for platelet and leucocyte counts, blood samples were drawn for routine hemogram at the time of admission. This was evaluated by fully automated haematology analyser-5 part differential. Thrombocytopenia was defined as platelet counts less than $1,50,000 /$ microliter and confirmed on peripheral smear examination (Figure 4).

Leucocyte counts of dengue patients were recorded and in majority of patients they were in normal range (4000-11000 per microliter) (Figure 5). Male preponderance was seen among positive cases (Figure 6).

DEN is an emerging vector- borne disease. Rapid urbanisation, globalisation, poor solid waste and water management and increasing population have given rise to new habitats for mosquito breeding thereby increasing the number of cases.

Dengue viruses are arboviruses capable of infecting humans and causing outbreaks. Dengue infection can be caused by any one or more of the four different but closely related serotypes; DEN1, DEN2, DEN3 or DEN4 dengue virus of the genus Flavivirus (WHO, 2011). Dengue fever (DF) is a self-limiting disease in majority of cases, rarely it may cause Dengue Hemorrhagic Fever (DHF) and
Dengue Shock Syndrome (DSS) (National Guidelines for Clinical Management of Dengue Fever, 2015). DF has been defined as fever with two or more symptoms including headache, retro-orbital pain, myalgia or arthralgia and leucopenia, thrombocytopenia. There are four grades of severity of DHF. DHF -I include above criteria for DF plus positive tourniquet test, evidence of plasma leakage with platelet count less than 100,000/ cu.mm and haematocrit rise $20 \%$ or more.

DHF II includes DHF I plus some evidence of spontaneous bleeding in skin or other organs (black tarry stools, epistaxis, bleeding from gums, etc.) and abdominal pain. DHF III includes DHF II plus circulating failure (weak rapid pulse, pulse pressure $<20 \mathrm{~mm} \mathrm{Hg}$ or high diastolic pressure, hypotension with the presence of cold clammy skin and restlessness). DHF IV incudes profound shock with undetectable blood pressure or pulse and haematocrit rise more than $20 \%$. DHF III and IV are the criteria for labelling as DSS (National Guidelines for Clinical Management of Dengue Fever, 2015).

Early diagnosis may be challenging as dengue infection presents with non-specific signs and symptoms which may not be easily differentiated from other febrile illnesses. In our study, peak incidence of dengue occurred in the month of October and November which correlates with other Indian studies (Chandy et al., 2013; Neeraja et al., 2006). This may be supported by scientific evidence of temperature and rainfall influencing dengue incidence. According to IDSP data, dengue fever cases peak during the post monsoon period between mid-September to November (National Centre for Disease Control Newsletter, 2013). The density of Aedes aegypti in an area is also linked with rainfall and water storage. Life span of this vector is influenced by temperature and humidity. 
Table.1 Shows the serological distribution of these positive cases. Out of 395 positive samples, $240(61 \%)$ were positive for NS1 antigen and 82 (21\%) for anti-dengue IgM \& IgG antibodies

\begin{tabular}{|l|c|c|c|c|c|c|c|}
\hline \multicolumn{1}{|c|}{ Month } & NS1 & $\begin{array}{c}\text { NS1+ } \\
\text { IgM }\end{array}$ & $\begin{array}{c}\text { NS1 + } \\
\text { IgG }\end{array}$ & IgM & IgG & IgM + IgG & NS1+IgG+IgM \\
\hline Jan & 4 & & & & & 1 & \\
\hline Feb & 1 & & & & 1 & 1 & \\
\hline Mar & 1 & & & & 1 & & \\
\hline Apr & 1 & & & & & & \\
\hline May & & & & & 1 & & \\
\hline June & 1 & & & & & & \\
\hline July & 5 & 1 & & & 4 & 4 & \\
\hline Aug & 13 & 3 & 3 & 1 & 2 & 9 & 5 \\
\hline Sep & 36 & 2 & & 3 & 7 & 8 & \\
\hline Oct & 65 & 2 & & 6 & 3 & 18 & \\
\hline Nov & 80 & 1 & & 10 & 8 & 30 & 5 \\
\hline Dec & 15 & 1 & 1 & 2 & 2 & 12 & 1 \\
\hline
\end{tabular}

\begin{tabular}{|l|c|c|}
\hline Month & Positive & Negative \\
\hline Jan (12) & 5 & 37 \\
\hline Feb (15) & 3 & 12 \\
\hline Mar (18) & 2 & 16 \\
\hline Apr (13) & 1 & 12 \\
\hline May (14) & 1 & 13 \\
\hline June (13) & 1 & 12 \\
\hline July (34) & 14 & 20 \\
\hline Aug (97) & 34 & 63 \\
\hline Sep (263) & 61 & 202 \\
\hline Oct (310) & 94 & 216 \\
\hline Nov (421) & 134 & 287 \\
\hline Dec (141) & 48 & 93 \\
\hline
\end{tabular}


Distribution of positive samples

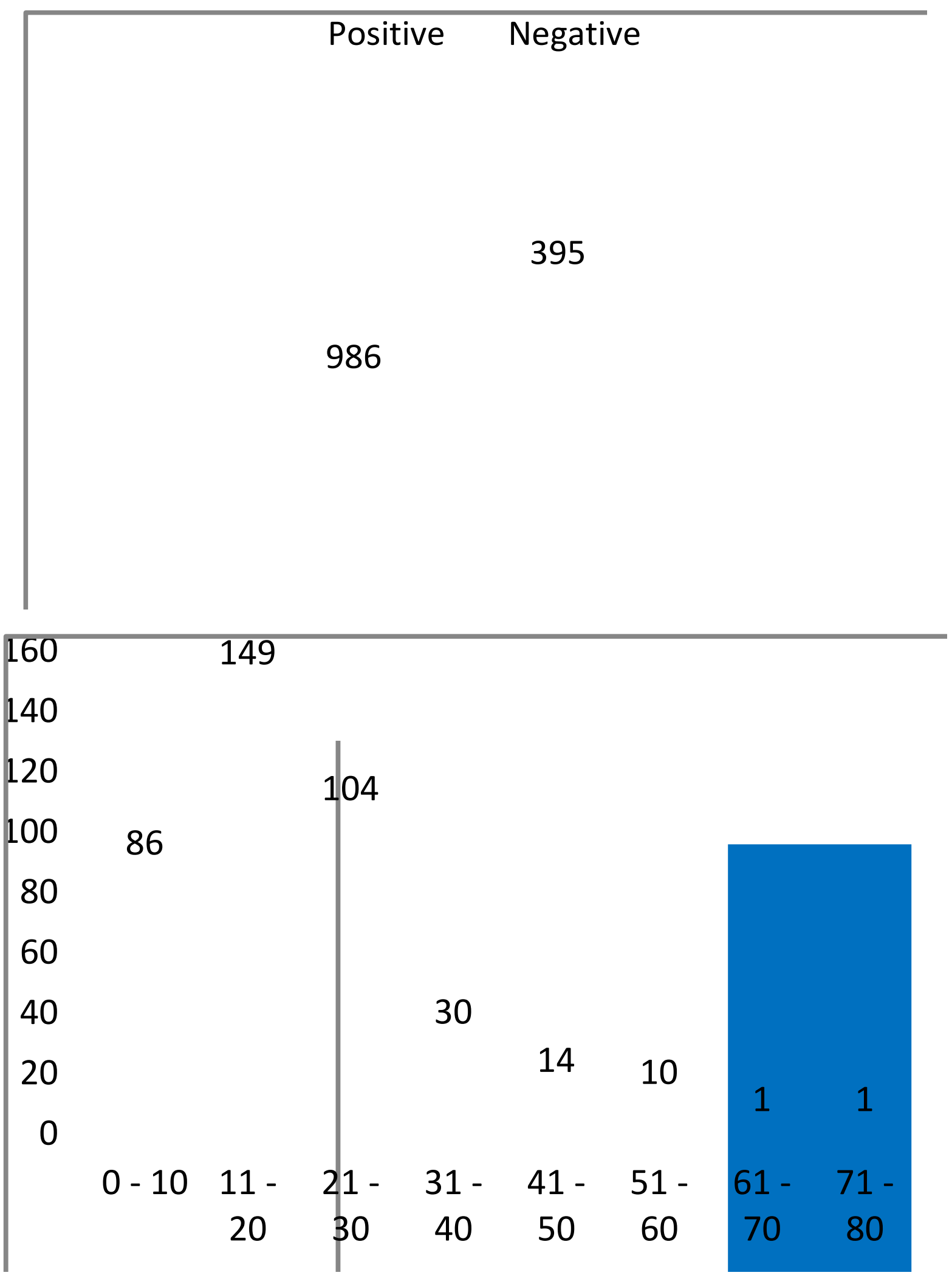



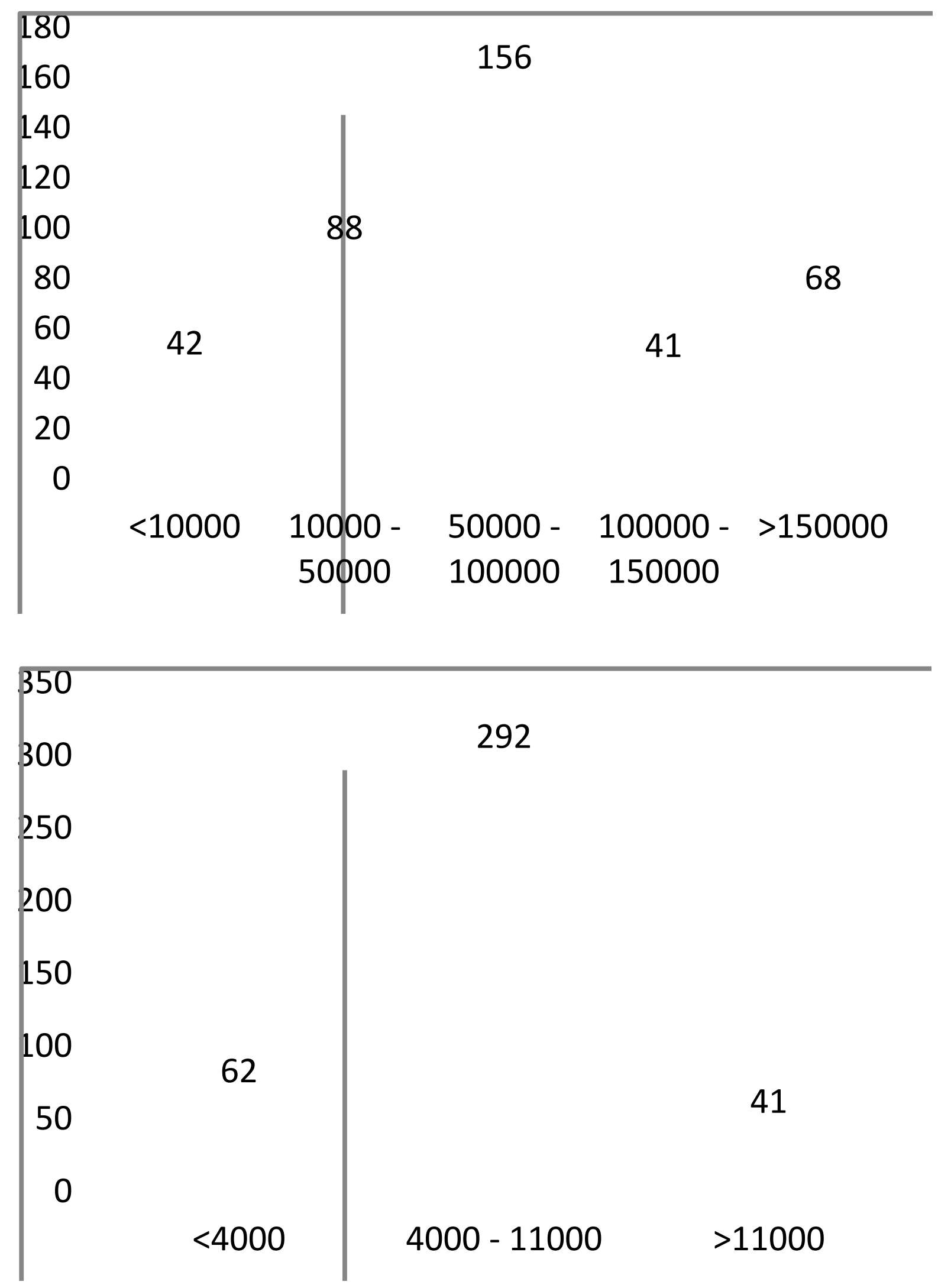
It survives best between $16^{\circ} \mathrm{C}-30^{\circ} \mathrm{C}$ and a relative humidity of $60-80 \%$; breeds in containers in and around the house. It has been observed that even a $2^{\circ} \mathrm{C}$ rise in temperature can reduce the extrinsic incubation period of DENV, hence an increase in mosquito density. The extent of dengue transmission depends on interplay of multiple factors including host population density, vector density and proportion of nonimmunized people in a community (Park, 2013; National Guidelines for Clinical Management of Dengue Fever, 2015; WHO, 2011; Chandy et al., 2013; Neeraja et al., 2006; National Centre for Disease Control Newsletter, 2013; Karim et al., 2012).

The association of the DEN positivity with thrombocytopaenia was in concordance with the findings of other recent Indian studies (Neeraja et al., 2013; Kulkarni et al., 2011). Majority of the cases in our study belong to the age group 20-40 years, with a male preponderance as seen in other studies (Kashinkunti et al., 2013; Chandralekha et al., 2008). Studies done by Halsey et al., and Kashinkunti et al., to correlate DEN with its clinical manifestations revealed that headache and myalgia/arthralgia were the most common clinical manifestation of DEN infection which was also seen in our study (Chandralekha et al., 2008; Halsey et al., 2012).

Diagnosis of dengue infection is confirmed by the detection of virus, viral genome or NS1 Antigen, or seroconversion of $\operatorname{IgM}$ or $\operatorname{IgG}$ (from negative to positive $\mathrm{IgM} / \mathrm{IgG}$ or fourfold increase in the specific antibody titre) in paired sera. Of all the methods available for dengue diagnosis, virus isolation provides the most specific test result. However, facilities that can support viral culture are not always available. The detection of the viral genome or viral antigens also provides evidence of infection. A large window of opportunity for Dengue diagnosis is provided by NS1 Ag which is highly conserved glycoprotein.

Single IgM ELISA test positivity is probable of dengue and definitive diagnosis requires use of paired sera to demonstrate rising titre (Halsey et al., 2012).

Majority of our cases $(61 \%)$ were detected exclusively by the presence of viral NS1 antigen compared to $\operatorname{IgM}(7 \%)$ antibodies in patient's sera. It is known that early detection of DEN cases by NS1 assay helps in diagnostic detection and confirmation of cases (Khan et al., 2014; Datta and Wattal, 2010). NS1 antigen detection is particularly useful during the first 5 days of illness and significantly more sensitive for primary than secondary DEN infection as was also seen in our study (Khan et al., 2014; Dussart et al., 2008; Lima Mda et al., 2010; Tricou et al., 2010). Five percent of NS1- positive samples were also IgG positive. These patients provided serological evidence of previous exposure. Fifteen patients who were simultaneously positive for NS1 antigen, IgM and $\mathrm{IgG}$ antibodies were probably in the late stage of either a primary or a secondary infection (Khan et al., 2014; Arya et al., 2011). Seven of these 15 patients had serological evidence of secondary infection. It is a known fact that during a primary infection, individuals develop IgM after 5-6 days and IgG antibodies after 7-10 days (Guzmán and Kourí, 2002; De Paula and Fonseca, 2004). Majority of the patients (71\%) presenting with fever of $>5$ days in our study were positive for IgM ELISA as compared to other serological parameters. In 2007, Kumarasamy et al., compared the use of NS1 ELISA with viral isolation in cell cultures and RT- PCR assay and achieved great results in patients in the early stages of infection concluding that NS1 antigen 
detection may be an appropriate marker of acute DENV infection (Kumarasamy et al., 2007). Thus, it can be stated that supporting clinical symptoms along with early detection of viral NS1 antigen can help speedup diagnosis of DEN cases during the first 5 days of fever and fever beyond that can be diagnosed by IgM ELISA alone.

\section{Limitations}

Molecular testing to know the serotype of dengue virus could not be done.

Dengue has become widespread and repeated attacks due to different serotypes are becoming common. Early diagnosis of dengue allows institution of appropriate supportive therapy and decreases risk of complications. In this regard, the role of the laboratory for diagnosis of Dengue, through serological, molecular and virological methods remains crucial to understand the exact burden of disease. Monitoring of climatic and environmental factors has important association with high vector density. Understanding of local epidemiology, risk factors and disease burden in dengue is essential to initiate preventive measures in time so that the outbreaks and epidemics can be avoided or controlled.

\section{References}

Arya SC, Agarwal N, Parikh SC, Agarwal S. Simultaneous detection of dengue NS1 antigen, IgM plus IgG and platelet enumeration during an outbreak. Sultan Qaboos Univ Med J 2011; 11: 470- 6.

Blacksell SD, Mammen MP Jr., Thongpaseuth S, Gibbons RV, Jarman $\mathrm{RG}$, Jenjaroen $\mathrm{K}$, et al., Evaluation of the Panbio dengue virus nonstructural 1 antigen detection and immunoglobulin M antibody enzyme- linked immunosorbent assays for the diagnosis of acute dengue infections in Laos. Diagn Microbiol Infect Dis 2008; 60: 43- 9 .

Chandralekha, Gupta P, Trikha A. The North Indian dengue outbreak 2006: A retrospective analysis of Intensive Care Unit admissions in a tertiary care hospital. Trans R Soc Trop Med Hyg 2008; 102: 143- 7.

Chandy S, Ramanathan K, Manoharan A, Mathai D, Baruah K (2013) Assessing effect of climate on the incidence of dengue in Tamil Nadu. Indian J Med Microbiol 31: 283-286.

Datta S, and Wattal C. Dengue NS1 antigen detection: A useful tool in early diagnosis of dengue virus infection. Indian J Med Microbiol 2010; 28: 107- 10.

De Paula SO, and Fonseca BA. Dengue: A review of the laboratory tests a clinician must know to achieve a correct diagnosis. Braz J Infect Dis 2004; 8: 390- 8 .

Dussart P, Petit L, Labeau B, Bremand L, Leduc A, Moua D, et al., Evaluation of two new commercial tests for the diagnosis of acute dengue virus infection using NS1 antigen detection in human serum. PLoS Negl Trop Dis 2008; 2: e280.

Guzmán MG, and Kourí G. Dengue: An update. Lancet Infect Dis 2002; 2: 33- 42.

Halsey ES, Marks MA, Gotuzzo E, Fiestas V, Suarez L, Vargas J, et al., Correlation of serotype- specific dengue virus infection with clinical manifestations. PLoS Negl Trop Dis 2012; 6: e1638.

Karim MN, Munshi SU, Anwar N, Alam MS (2012) Climatic factors influencing dengue cases in Dhaka city: A model for dengue prediction. Indian J Med Res 136: 32-39.

Kashinkunti MD, Shiddappa, Dhananjaya M. A study of clinical profile of dengue 
fever in a tertiary care teaching hospital. Sch J Appl Med Sci 2013; 1: 280- 2.

Khan SA, Dutta P, Topno R, Soni M, Mahanta J. Dengue outbreak in a hilly state of Arunachal Pradesh in Northeast India. Scientific World Journal 2014; 2014: 584093.

Kulkarni RD, Patil SS, Ajantha GS, Upadhya AK, Kalabhavi AS, Shubhada RM, et al., Association of platelet count and serological markers of dengue infection - .Importance of NS1 antigen. Indian J Med Microbiol 2011; 29: 359- 62.

Kumarasamy V, Wahab AH, Chua SK, Hassan Z, Chem YK, et al., Evaluation of a commercial dengue NS1 antigencapture ELISA for laboratory diagnosis of acute dengue virus infection. J Virol Methods 2007; 140: 75-79.

Lima Mda R, Nogueira RM, Schatzmayr HG, dos Santos FB. Comparison of three commercially available dengue NS1 antigen capture assays for acute diagnosis of dengue in Brazil. PLoS Negl Trop Dis 2010; 4: e738.

National Centre for Disease Control Newsletter (2013) Quarterly newsletter from the national centre for disease control (NCDC).

National Guidelines for Clinical Management of Dengue Fever (2015) National Vector Borne Disease Control
Programme, World Health Organization, India.

Neeraja M, Lakshmi V, Dash PK, Parida MM, Rao PV. The clinical, serological and molecular diagnosis of emerging dengue infection at a tertiary care institute in Southern, India. J Clin Diagn Res 2013; 7: 457- 61.

Neeraja M, Lakshmi V, Teja VD, Umabala P, Subbalakshmi MV (2006) Serodiagnosis of dengue virus infection in patients presenting to a tertiary care hospital. Indian J Med Microbiol 24: 280-282.

Park K (2013) Preventive and social medicine: Dengue syndrome (22nd edn). Banarasidas Bhanot, Jabalpur.

Tricou V, Vu HT, Quynh NV, Nguyen CV, Tran HT, Farrar J, et al., Comparison of two dengue NS1 rapid tests for sensitivity, specificity and relationship to viraemia and antibody responses. BMC Infect Dis2010; 10: 142.

WHO (2011) Comprehensive guidelines for prevention and control of dengue and dengue hemorrhagic fever, revised and expanded edition, SEAR.

World Health Organization. Dengue: Guidelines for Diagnosis, Treatment, Prevention and Control. 2nd ed. Geneva: World Health Organization; 2009. p. 1- 144.

\section{How to cite this article:}

Qursheed Sultana, Mohammed Abdur Rab Ansari, Mahwish Jawaid, Mohd Ahmed, Khaled Ahmed and Maimoona Mustafa. 2018. Clinical, Epidemiological and Laboratory Profile of Dengue Fever at Tertiary Care Hospital in Hyderabad, India. Int.J.Curr.Microbiol.App.Sci. 7(10): 2496-2504. doi: https://doi.org/10.20546/ijcmas.2018.710.289 